\title{
Dentist-patient communication: what do patients and dentists remember following a consultation? Implications for patient compliance
}

This article was published in the following Dove Press journal:

Patient Preference and Adherence

14 June 2013

Number of times this article has been viewed

\author{
Sara Misra \\ Blánaid Daly \\ Stephen Dunne \\ Brian Millar \\ Mark Packer \\ Koula Asimakopoulou \\ Dental Institute, King's College \\ London, London, UK
}

Correspondence: Koula Asimakopoulou KCL Dental Institute, Unit of Social and Behavioural Sciences, Bessemer Road, Denmark Hill, London SE5 9RW, UK Tel +44203299348I

Email koula.asimakopoulou@kcl.ac.uk
Background: There is a lack of information about the extent to which patients recall key facts of dental consultations. Forgetting health advice undermines adherence with such instructions and is a potential problem. This study assessed the quantity and type of information recalled in a dental consultation, dentist-patient agreement over the contents of the consultation, and the relationship of such recall with patient satisfaction.

Methods: Using a cross-sectional design, questionnaire data were obtained from patients recruited through a letter and presenting for a routine dental consultation. General issues discussed, specific information about oral health given, dentist-performed procedures, and agreed future actions were reported independently in writing, by patients and also by the treating dentist immediately postconsultation. Additionally, patients completed a dental visit satisfaction questionnaire.

Results: Responses ( $n=26,55 \%$ response rate) were content-analyzed, and data on the number and type of information that was recalled were obtained. Interrater reliability was established. Inferential testing showed differences in dentist-patient recall, dentist-patient agreement, and the association between patient recall and satisfaction. Dentists recalled more information than patients $(P=0.001)$. Dentists further reported giving more dental health education $(P=0.006)$ and discussing more future actions $(P=0.002)$ than patients actually remembered. Technical (eg, crowns/bridges) rather than psychosocial (eg, pain/embarrassment) issues were reported more often $(P=0.001)$ by both dentists and patients. Dentist-patient agreement over issues discussed and procedures performed was higher (kappa $=0.210-0.310)$ than dental health education agreement and agreed future actions (kappa $=0.060-0.110)$. There was no relationship between patient recall and patient satisfaction with the consultation $(P=0.240)$.

Conclusion: Patients do not recall as much advice and agreed actions about future dental care as dentists believe they have discussed. These results have implications for patient adherence with oral health instructions.

Keywords: patient memory, dental consultation, patient satisfaction

\section{Introduction}

Ley's seminal work ${ }^{1,2}$ proposed that adherence with health advice is a function of three patient-related factors: satisfaction, understanding, and memory. Specifically, he theorized that patient understanding and memory for the consultation have direct effects on adherence with medical advice, but also indirect effects through influencing patient satisfaction.

Since then, a lot of work has taken place in the area of patient recall. It is now well established that patient recall of a medical consultation is limited, with patients 
forgetting $40 \%-80 \%$ of information given. ${ }^{3}$ This observation seems to hold true in various medical settings. For instance, in a study of children's immunization, the majority of mothers were unable to name the immunization their child had received, immediately after leaving the treatment room. ${ }^{4}$ Similarly, $70 \%$ of patients who had recently undergone laparoscopic surgery were unable to recall a single risk factor to do with the procedure, 5 days following patient receipt of this information and consent to surgery. ${ }^{5}$

At the same time, in dentistry there is limited evidence about the quantity of information recalled postconsultation. For example, in a study examining orthodontic patients' information retention, around $70 \%$ of information conveyed could not be recalled 10 days later. ${ }^{6}$ Similarly, $40 \%$ of oral surgery patients failed to remember receiving written postoperative instructions, a finding that might be related to the fact that $67 \%$ of these participants failed to comply with antibiotic prescriptions. ${ }^{7}$ It appears that memory for specific, posttreatment information in dental settings is problematic.

Research has focused on patient memory for very specific, dental treatment-related information rather than any other aspect of the consultation. The question as to what sort of information (rather than how much) is recalled following a dental consultation remains unanswered. We know that, generally, recall is influenced by people's prior knowledge and expectations, ${ }^{8-11}$ so some aspects of the consultation that the patient expects to experience in the consultation might be better recalled than others.

Studies reporting on patient-health-care professional (HCP) concordance over the contents of routine visits show that concordance can be poor, with patients and HCPs disagreeing on the most basic of issues, such as whether the doctor had in fact provided any treatment at all. ${ }^{12}$ Similarly, in a study where HCPs and their patients recalled the key issues discussed, decisions made, and set goals, immediately after a routine outpatient diabetes consultation HCPs and patients disagreed about issues discussed and decisions made $20 \%$ of the time. The extent of the disagreement was double this frequency when recalling set goals. The authors concluded that ". . . the two parties concerned seemed to recall different consultations." ${ }^{13}$ Further work on concordance between GPs and their patients showed that patients' and GPs' perceptions regarding consultation content were discordant $60 \%$ of the time. ${ }^{14}$ It would appear then that in some cases the information that HCPs believe they have communicated might not be the same as what patients believe they have heard.

A similar lack of concordance was reported in a concurrent community dental survey, where although $41 \%$ of dentists recalled having advised smoking patients to quit, only $7 \%$ of smokers in that same community reported having received that advice during dental visits. ${ }^{15}$

These findings have implications for patient adherence and patient satisfaction following a dental consultation. If patients fail to recall basic information given at dental consultation, their efforts to adhere to it will be undermined. Equally, where recall of a consultation is a predictor of satisfaction, ${ }^{1,2}$ satisfaction with the consultation may be undermined.

Whilst dental patient memory for behaviors such as smoking cessation may be low, the question as to whether patients remember any of the contents of a routine dental assessment consultation remains unanswered. Therefore, this study had the following objectives. These were to examine:

- dentist-patient differences in the quantity and type of material recalled

- dentist-patient recall concordance

- the relationship between patient recall of and satisfaction with the consultation.

We hypothesized that there would be differences between dentists' and patients' recall of the consultation, that there would be weak recall concordance between dentists and patients, and that there would be a relationship between patient recall and patient satisfaction. The null hypotheses for the above were that there would be no differences in recall between patients and dentists, no recall concordance between patients and dentists, and no relationship between patient recall and satisfaction.

\section{Materials and methods}

In a cross-sectional survey design, dentists and patients selfreported the contents of a dental consultation immediately after the consultation. Patients also reported on satisfaction with the visit. The consultation involved an oral examination and discussion of the patient's oral health problem.

A demographic information questionnaire asked for patients' sex, age-group, and marital and employment status. Patients further self-reported using the reliable (Cronbach's $\alpha=0.92$ ) dental visit satisfaction questionnaire, ${ }^{16}$ scored on a 5-point Likert scale, and also a consultation-recall measure, with the wording slightly adapted from previous work ${ }^{13}$ for use in a dental setting. This measure asked patients and dentists to list the issues they discussed, any information patients were given about their dental health, the dental procedures performed at consultation, and any actions the dentist and the patient had agreed upon. Standard participant information and consent forms were also used. 
Permission to undertake this study was obtained from a National Health Service ethics committee and the King's College London Hospital Research and Development bodies, in the UK.

All dental patients over 18 years old who could read, write, and understand English, who were able to give consent, and were due to attend a routine assessment in the department of primary dental care at the King's College Hospital campus of King's College London Dental Institute between February and May 2010 were eligible to participate.

Patients fulfilling the inclusion criteria were invited to participate by letters giving information about the study, sent in advance of their appointment. No incentives were offered to patients. Invited patients were then made known to the researcher via the clinic receptionist. These patients were approached by the researcher. Of those approached $(n=47)$, $26(55 \%)$ agreed to be included in the study. Of those who declined participation, reasons cited for doing so included lack of time (27\%), further appointments with another HCP (20\%), and dental pain (20\%). Patients were assured of anonymity and confidentiality, and completed a consent form having been given the opportunity to ask questions about the study.

The predominantly single (single $/$ married $=15 / 6$, four not disclosed), female (female/male $=15 / 11$ ), not currently employed $($ employed/unemployed $=11 / 15)$ patient sample was older ( 16 aged over 40 years, 7 aged $30-40$ years, 3 aged 20-30 years). The 26 dental consultations were all carried out by two experienced white male consultant dental practitioners in their 50s who were blind to the study hypotheses. The dentists had no preset formats to follow, but were instead carrying out routine, nonemergency, dental hospital initial assessment consultations designed to identify the patient's oral health issue and prescribe the most appropriate dental treatment.

Within 2-5 minutes of the end of the dental consultation both treating dentist and patient completed the consultationrecall measure. Only the patients completed the dental visit satisfaction questionnaire. Individual pen-and-paper questionnaire completion took no longer than 5 minutes for dentists and patients alike; the anonymized forms were returned to the researcher at the end of the clinic.

\section{Statistical analyses}

Dentist and patient questionnaire responses were inspected and content-analyzed along the following recall categories $(\mathrm{C})$ :

- C1: General issues discussed

- C2: Specific information offered about oral health (health education)
- C3: Procedures performed by the dentist

- C4: Actions agreed on between dentist and patient.

The overall number of activities (sum of $\mathrm{C} 1-\mathrm{C} 4$ ) reported as having taken place in the consultation was also recorded.

Two of the authors (SM and KA) inspected all questionnaires and extracted data independently. Standard exploratory conceptual content-analysis procedures were adhered to throughout, where each line of text was coded for content using exclusive and exhaustive units of analysis. ${ }^{17}$ Interrater agreement was high, both for dentists' (84\%) and patients' $(87 \%)$ responses.

$t$-Tests assessed differences in recall between patients and dentists, separately for each recall category (C1-C4) and overall for the whole consultation. They were also used to assess differences between dentists and patients in the type of issues they recalled and also within groups to assess whether each recalled more psychosocial rather than technical issues. An issue was considered technical if it involved a procedure that could only be completed by a trained dentist. All other issues to do with emotions, beliefs, and pain were coded as psychosocial.

Kappa coefficients were used to assess recall agreement between dentists and patients. Agreement was calculated individually for each single topic recorded in the recall questionnaires. The level of agreement for each questionnaire category $(\mathrm{C} 1-\mathrm{C} 4)$ was by one of two scores: where the issues identified by dentists and patients were the same or broadly the same, the data were treated as being in agreement, and a score of 1 was allocated to responses. Where there was no overlap whatsoever between responses, and a topic was mentioned by either dentist or patient only, data were coded as being in disagreement, and this disagreement was coded as 0 .

Pearson's correlation assessed the association between patient recall and satisfaction with the consultation.

The study had $80 \%$ power to detect a large, clinically relevant effect size $(d=0.80)$ at a $95 \%$ confidence level and assuming two-tailed independent group comparisons. SPSS version 17 (IBM, Armonk, NY, USA) was used for all analyses.

\section{Results}

Quantity of material recalled by dentists and patients

Data for each aspect of the consultation were collated and analyzed. The findings (mean, standard deviation [SD], and example activities reported), appear in Table 1. 
Table I Dentist and patient recall data $(n=26)$ by questionnaire response category $(\mathrm{C})$

\begin{tabular}{|c|c|c|c|}
\hline & Dentist & Patient & $P$-value \\
\hline & $\overline{\text { Mean (SD) }}$ & $\overline{\text { Mean (SD) }}$ & \\
\hline $\begin{array}{l}\text { CI: general issues } \\
\text { discussed } \\
\text { (eg, problems with tooth } \\
\text { erosion, problems with } \\
\text { veneers, inflamed gums, } \\
\text { denture issues, sore tongue) }\end{array}$ & $\begin{array}{l}2.880 \\
(0.760)\end{array}$ & $\begin{array}{l}2.230 \\
(1.470)\end{array}$ & 0.06 \\
\hline $\begin{array}{l}\text { C2: dental health advice } \\
\text { (eg, brushing thoroughly, } \\
\text { flossing technique, advice } \\
\text { to stop smoking, when } \\
\text { to remove dentures) }\end{array}$ & $\begin{array}{l}1.420 \\
(0.810)\end{array}$ & $\begin{array}{l}0.850 \\
(0.680)\end{array}$ & 0.007 \\
\hline $\begin{array}{l}\text { C3: procedures } \\
\text { performed } \\
\text { (eg, mouth examination, } \\
\text { replaced crown, polished } \\
\text { teeth, took impressions) }\end{array}$ & $\begin{array}{l}\text { I.460 } \\
(0.650)\end{array}$ & $\begin{array}{l}1.500 \\
(0.760)\end{array}$ & 0.845 \\
\hline $\begin{array}{l}\text { C4: agreed actions } \\
\text { (eg, refer to different } \\
\text { clinic, book follow-up, } \\
\text { bring mouth guard to next } \\
\text { appointment) }\end{array}$ & $\begin{array}{l}2.310 \\
(0.970)\end{array}$ & $\begin{array}{l}1.350 \\
(1.090)\end{array}$ & 0.002 \\
\hline $\begin{array}{l}\text { Overall consultation } \\
\text { activities }\end{array}$ & $\begin{array}{l}8.080 \\
(1.740)\end{array}$ & $\begin{array}{l}5.920 \\
(2.620)\end{array}$ & 0.001 \\
\hline
\end{tabular}

Abbreviation: SD, standard deviation.

Across 26 consultations, it is evident that with the exception of procedures performed (C3), where both dentists' and patients' recall levels appeared similar at approximately 1.5 procedures per consultation $(t[50]=0.19, P=0.845)$, and the general issues discussed $(\mathrm{C} 1)$, where both patients and dentists reported an average of two issues per consultation $(t[50]=2.003, P=0.060)$, there were significant differences in health advice given (C2) and agreed-upon actions (C4). Dentists reported having given approximately twice more dental health advice $(t[50]=2.78, P=0.007)$ than the advice patients recalled. Dentists also reported having agreed twice more future actions $(t[50]=3.35, P=0.002)$ than patients noted. When examining the sum of activities reported for the consultation as a whole, dentists' recall was significantly different to that of patients $(t[50]=3.49, P=0.001)$, with dentists reporting more activities (mean $=8.08, \mathrm{SD}=1.74)$ taking place per consultation than patients ( mean $=5.92, \mathrm{SD}=2.62$ ).

\section{Are there differences in information content that dentists and patients remember?}

The issues found in dentists' and patients' responses were coded as to whether they were psychosocial or technical.
Table 2 presents descriptive and inferential statistics on dentists' and patients' recall of these issues.

Dentists $(t[25]=14.35, P=0.001)$ and patients $(t[25]=7.40, P=0.001)$ reported seven and five times more technical than psychosocial details, respectively, about the consultation. Examination of each group separately revealed dentists recalled almost twice more technical concepts than patients $(t[50]=3.824, P=0.001)$, but there was no difference between dentists and patients in the few psychosocial issues they each reported $(t[50]=0.64, P=0.530)$.

\section{What is the extent of agreement in recalled issues between dentists and patients?}

The degree of agreement between dentist and patient for each item and overall was analyzed using the kappa coefficient and the Landis and Koch ${ }^{18,19}$ interpretation of these values. These results are shown in Table 3.

Kappa values for items $\mathrm{C} 1$ and $\mathrm{C} 3$ were moderate $(0.310$ and 0.210 respectively) suggesting only fair agreement between dentists and patients on the issues discussed and procedures performed in the consultation. For categories $\mathrm{C} 2$ and C4 (dental health education and decisions agreed on), the kappa values indicated weak concordance (at 0.060 and 0.110 , respectively).

\section{Is there a relationship between patient recall of the consultation and patient satisfaction?}

Dental satisfaction questionnaire responses could range from 10 to 50 . Higher scores indicated higher dissatisfaction. Patients were highly satisfied with the consultation $($ mean $=12, \mathrm{SD}=3)$. The relationship between patient satisfaction and patient recall of the consultation was not significant $(r[24]=-0.24, P=0.240)$.

Table 2 Dentist and patient recall data $(n=26)$ by content (psychosocial vs technical)

\begin{tabular}{|c|c|c|}
\hline Patient & Dentist & Patient-dentist \\
\hline Mean (SD) & Mean (SD) & $\begin{array}{l}\text { Mean difference } \\
(P \text {-value })\end{array}$ \\
\hline \multicolumn{3}{|l|}{ Psychosocial issues } \\
\hline $1.040(0.960)$ & $0.880(0.770)$ & $0.160(0.530)$ \\
\hline \multicolumn{3}{|l|}{ Technical issues } \\
\hline $4.880(2.450)$ & $7.190(1.860)$ & $-2.310(0.001)$ \\
\hline \multicolumn{3}{|l|}{ Technical/psychosocial } \\
\hline Mean difference ( $P$-value) & $3.84(0.00 \mathrm{I})$ & $6.3 \mathrm{I}(0.00 \mathrm{I})$ \\
\hline
\end{tabular}

Abbreviation: $S D$, standard deviation. 
Table 3 Dentist and patient agreement data $(n=26)$ by questionnaire response category $(\mathrm{C})$

\begin{tabular}{llll}
\hline Category & Kappa & Standard error & P-value \\
\hline $\mathrm{Cl}$ : issues discussed & 0.310 & 0.080 & 0.001 \\
$\mathrm{C} 2$ : dental health advice & 0.060 & 0.120 & 0.310 \\
$\mathrm{C} 3$ : procedures performed & 0.210 & 0.140 & 0.060 \\
C4: agreed actions & 0.110 & 0.090 & 0.001 \\
Overall & 0.250 & 0.050 & 0.001 \\
\hline
\end{tabular}

\section{Discussion}

This exploratory study addressed several questions. Firstly, we examined whether there are differences in the quantity of information recalled postconsultation between dentists and patients. Secondly, we explored the recalled material in terms of content, and explored whether dentists and patients recall psychosocial and technical aspects of the consultation differently. Thirdly, we evaluated the extent of disagreement between dentists and patients on the content of the consultation. Finally, we investigated the relationship between consultation recall and patient satisfaction.

Overall, dentists' recall was greater than that of patients. So not only did dentists recall more activities taking place in the consultation overall, but specifically they appeared to recall more oral health advice being offered and more discussion of follow-up actions. Patients, on the other hand, remembered a similar number of performed procedures and general issues, but were particularly poor at recalling dental health advice and future actions relating to the consultation.

These results are not wholly unexpected. One reason why dentists' recall seems superior to that of patients could be that dentists have overall control of the consultation and its structure. ${ }^{20}$ As such, they are probably better placed to recall content, as they are more experienced with the consultation setup and the context, being primarily responsible for both. Bartlett's ${ }^{21}$ seminal work on the use of context as an aid to recall fully explains these findings. Future work should vary the communication model under which dentists work to one where, for example, patients are primarily in control of and drive the communication during a consultation and then reassess recall in this context. Such a patient-led consultation would see the length, quality, and quantity of the interaction during the consultation suggested and driven by the patient, with the dentist responding to patient suggestions rather than taking a lead themselves, in line with Szasz and Hollender's ${ }^{22}$ "patient as expert" model of communication.

Patients, on the other hand, were no different to dentists in recalling the main issues discussed and procedures performed.
We suggest that both of these aspects of the consultation relate to the "here and now"; patients remember what brought them to see the dentist and what the dentist did to their mouth at that point. Their recall, however, of any dental health advice given in order to deal with their condition and any agreed-upon actions - both concepts relating to future adherent behavior - was poor. These findings extend previous work in medical ${ }^{13}$ and dental ${ }^{15}$ settings by showing that patients remember some aspects of what is discussed, but apparently not those aspects that are likely to help their oral health, such as dental health advice and future actions. These findings have worrying implications for patient adherence and go some way towards explaining the substantial rates of patient nonadherence with HCP instructions reported in the literature. ${ }^{23}$ Patients are unlikely to follow health advice and instructions if they have not registered these in the first place. Where in the consultation such future advice is given should also be examined in future work.

Interestingly, both patients and dentists recalled more technical than psychosocial aspects of the consultation. There are several explanations for this finding: firstly, it could be that the style of the dental consultation is geared towards emphasizing technical procedures rather than patients' psychosocial problems, such as embarrassment and worry. In line with a biomedical model where dentists, constrained by the amount of time, expertise, and resources available, need to focus on fixing dental problems rather than dealing with patients' feelings about them, it is not surprising that they reported such aspects of the consultation more often than patients' psychosocial problems. Similarly, being treated in an environment where technical procedures rather than psychosocial talk (or talk of any kind) dominate the consultation, it is not surprising that these aspects of the consultation were more salient for patients too. A further plausible explanation has to do with patient expectations: where patients expect the dentist to perform technical procedures rather than deal with their emotional problems arising from these, patients' recall was in line with their expectations..$^{8-11}$ Finally, it could be that a stronger emotional response was associated with the oral health issues discussed and procedures performed (both of which might be associated with pain and anxiety), whereas discussions about preventive oral health care might not have aroused much in the way of an emotional response. Emotion-provoking events are remembered more vividly (although not necessarily more accurately), which may explain these findings. ${ }^{24}$ Either way, to the extent that recalling dental technical procedures may be necessary for future patient adherence, patients in our study seemed to be remembering these clearly. 
Dentist-patient agreement over consultation content supported previous work. ${ }^{12-14}$ Patients' and dentists' recollections of discussions were discordant in terms of the dental health advice given and future actions that were agreed upon. These results support an Australian study on smoking cessation that found dentists believing they had advised smoking cessation, but patients not recalling such advice ever having been given. ${ }^{15}$ The implications of this work for patient adherence to a health-care plan would lead us to assume that it is very likely that advice will not be followed. The reasons for this discrepancy may be that dentists had discussed oral health advice, but patients were not able to remember it. Alternatively, it could be that dentists had it in mind to discuss these issues, but never quite did, or presented the information in a way that the patient did not understand or register. Future work should involve the assessment of an audio record of consultations to evaluate dentists' and patients' recall with reference to what was actually said at consultation. Either way, a patient who is unable to recall oral health advice or future actions at consultation is unlikely to be in a position to act on such advice. Patients should perhaps be given in writing a list of advice given and actions agreed upon at a consultation so that such information might aid subsequent recall and adherence in future. ${ }^{25}$ It is suggested that patients are unlikely to adhere to advice they cannot recall immediately after leaving the consultation, and to this end patient memory and understanding of consultation instructions needs to be evaluated.

Finally, our sample reported high levels of satisfaction with the dental consultation. Satisfaction, however, was completely unrelated to recall of the consultation. This contradicts Ley's hypothesis on the importance of memory for patient satisfaction. ${ }^{1}$ Today's patients are probably quite different to those studied by Ley in the 1960s, with issues other than the details of the consultation influencing their satisfaction. So it could be that the patient's personal relationship with the dentist, the dentist's technical competence, the facilities present, and convenience factors ${ }^{20,26}$ are better predictors of patient satisfaction in the dental surgery, rather than memory. Alternatively, given that satisfaction was highly skewed in this study, the lack of a relationship may have resulted from a ceiling effect on the satisfaction variable.

This was a small exploratory study, which although sufficiently powered to detect differences between conditions, used a small sample of participants from one dental hospital. Future work needs to be undertaken to replicate these findings in different dental settings and at a different time period, perhaps introducing time delay postconsultation before questionnaires are completed. It is acknowledged that the low overall recall of patients in this study could have been responsible for the low recall across categories that were observed; future studies should replicate our work to confirm that the category-specific differences we have found here are not the results of overall floor effects. It is also suggested that future work should assess and control for levels of patients' dental anxiety and current pain, in that both these variables may affect recall. Finally, like any work relying on self-reports, the present study may well have suffered from social desirability effects, with patients and dentists selfreporting what they felt we wanted to see, rather than what actually happened. The fact that both dentists and patients were blind to the study hypotheses however, goes some way towards minimizing such effects.

\section{Conclusion}

This study has shown that dentists' recall of the consultation is different to that of patients. Worryingly, patients seem unable to recall accurately future dental health advice or agreed actions, and assuming this information has been communicated by dentists in the first place, this finding has implications for patient adherence to advice given at dental consultations. In order to support patient adherence in dental settings, measures need to be taken in practice to ensure that patients remember consultation advice immediately postconsultation.

\section{Acknowledgment}

The authors would like to thank all patients who took part in this study.

\section{Disclosure}

The authors report no conflicts of interest in this work.

\section{References}

1. Ley P. Memory for medical information. Br J Soc Clin Psychol. 1979; 18(2):245-255

2. Ley P. Doctor-patient communication: some quantitative estimates of the role of cognitive factors in non-compliance. J Hypertens Suppl. 1985; 3(1):S51-S55.

3. Kessels RP. Patients' memory for medical information. J R Soc Med. 2003;96(5):219-222.

4. Lee L, Brittingham A, Tourangeau R, et al. Are reporting errors due to encoding limitations or retrieval failure? Surveys of child vaccination as a case study. Appl Cogn Psychol. 1999;13(1):43-63.

5. Kriwanek S, Armbruster C, Beckerhinn P, Blauensteier W, Gschwantler M. Patients' assessment and recall of surgical information after laparoscopic cholecystectomy. Dig Surg. 1998;15(6):669-673.

6. Witt E, Bartsch A. The effect of information and communication in the orthodontic consultation. 1. The imparting of the information. Fortschr Kieferorthop. 1993;54(5):187-195. 
7. Blinder D, Rotenberg L, Peleg M, Taicher S. Patient compliance to instructions after oral surgical procedures. Int J Oral Maxillofac Surg 2001;30(3):216-219.

8. Oswald M, Grosjean S. Confirmation bias. In: Pohl RF, editor. Cognitive Illusions: A Handbook of Fallacies and Biases in Thinking, Judgement and Memory. Hove: Psychology Press; 2004:79-96.

9. Stangor C, McMillan D. Memory for expectancy-congruent and expectancy-incongruent information: a review of the social and social developmental literatures. Psychol Bull. 1992;111(1):42-61.

10. Kunda Z. Social Cognition: Making Sense of People. Massachussets: MIT Press; 1999.

11. Sanitioso R, Kunda Z, Fong GT. Motivated recruitment of autobiographical memories. J Pers Soc Psychol. 1990;59(2):229-241.

12. Rohrbaugh M, Rogers JC. What did the doctor do? When physicians and patients disagree. Arch Fam Med. 1994;3(2):125-128; discussion 129.

13. Parkin T, Skinner TC. Discrepancies between patient and professionals recall and perception of an outpatient consultation. Diabet Med. 2003;11:909-914.

14. Fagerberg CR, Kragstrup J, Stovring H, Rasmussen NK. How well do patient and general practitioner agree about the content of consultations? Scand J Prim Health Care. 1999;17(3):149-152.

15. Brink SG, Gottlieb NH, McLeroy KR, Wisotzky M, Burdine JN. A community view of smoking cessation counseling in the practices of physicians and dentists. Public Health Rep. 1994;109(1):135-142.

16. Corah NL, O'Shea RM, Pace LF, Seyrek SK. Development of a patient measure of satisfaction with the dentist: the Dental Visit Satisfaction Scale. J Behav Med. 1984;7(4):367-373.
17. Krippendorf K. Content Analysis: An Introduction to Its Methodology, 2nd ed. London: Sage; 2004.

18. Landis JR, Koch GG. An application of hierarchical kappa-type statistics in the assessment of majority agreement among multiple observers. Biometrics. 1977;33(2):363-374.

19. Landis JR, Koch GG. The measurement of observer agreement for categorical data. Biometrics. 1977;33(1):159-174.

20. Newsome PR, Wright GH. A review of patient satisfaction: 1. Concepts of satisfaction. Br Dent J. 1999;186(4 Spec No):161-165.

21. Bartlett F. Remembering. Cambridge: Cambridge University Press; 1932.

22. Szasz TS, Hollender MH. A contribution to the philosophy of medicine: the basic models of the doctor-patient relationship. AMA Arch Intern Med. 1956;97(5):585-592.

23. DiMatteo MR. Variations in patients' adherence to medical recommendations: a quantitative review of 50 years of research. Med Care. 2004;42(3):200-209.

24. Conway MA. Autobiographical Memory: An Introduction. Philadelphia: Open University Press; 1990.

25. Thickett E, Newton JT. Using written material to support recall of orthodontic information: a comparison of three methods. Angle Orthod. 2006;76(2):243-250.

26. Newsome PR, Wright GH. A review of patient satisfaction: 2. Dental patient satisfaction: an appraisal of recent literature. Br Dent J. 1999; 186(4 Spec No):166-170.
Patient Preference and Adherence

\section{Publish your work in this journal}

Patient Preference and Adherence is an international, peer-reviewed, open access journal focusing on the growing importance of patient preference and adherence throughout the therapeutic continuum. Patient satisfaction, acceptability, quality of life, compliance, persistence and their role in developing new therapeutic modalities and compounds to

\section{Dovepress}

optimize clinical outcomes for existing disease states are major areas of interest. This journal has been accepted for indexing on PubMed Central. The manuscript management system is completely online and includes a very quick and fair peer-review system. Visit http://www.dovepress.com/ testimonials.php to read real quotes from published authors. 